\title{
Editorial: Multimodality Molecular Imaging
}

\author{
Zhen Cheng $^{1 *}$ and Gerard Marriott ${ }^{2}$ \\ ${ }^{1}$ Molecular Imaging Program at Stanford, Department of Radiology and Bio-X Program, Canary Center at Stanford for \\ Cancer Early Detection, Stanford University, Stanford, CA, United States, ${ }^{2}$ Department of Bioengineering, University of \\ California, Berkeley, Berkeley, CA, United States
}

Keywords: CT, imaging probe, optical imaging, MRI, PET, multimodality imaging

\section{Editorial on the Research Topic}

\section{Multimodality Molecular Imaging}

Molecular imaging technologies are an essential component of precision medicine-based approaches to healthcare. In recent years, powerful imaging tools have been developed to detect diseases at an early stage of their development, to assess patients prognosis, stratification and monitoring treatments, and for image-guided therapy. These applications may be improved by using probes that allow for multimodal molecular imaging, in particular, by combining different types of imaging modalities that simultaneously furnish information on multiple physiological and anatomical aspects of diseases. Analysis of these high-content image data would assist physicians to diagnose better and treat diseases.

In this Research Topic, we focus on new developments in multimodal imaging technologies and their applications to precision medicine, including studies on multimodality imaging probes, instruments, and image analysis written by leading researchers in the field. In particular, Liu et al. describe the biodistribution and radiation dosimetry of a novel positron emission tomography (PET) probe, ${ }^{64} \mathrm{Cu}$ labeled BaBaSar-RGD2, in a non-human primate model. Liu et al. have extended earlier studies of this PET probe to image integrin $\alpha \mathrm{v} \beta 3$-targeted PET imaging of tumors relevant to clinical medicine. In a second research article McDougald et al. investigated two critical issues in PET/computed tomography (CT) imaging, namely, the effects of different CT acquisition protocols on PET/CT image quality and data quantification, and the absorbed dose associated with different CT parameters. The results reported here will be helpful in the design of protocols for small animal PET/CT imaging studies.

The first review article by Cal-Gonzalez et al., Cal-Gonzalez et al. describes the development of a variety of hybrid imaging systems such as SPECT/CT, PET/CT, and PET/MRI for pre-clinical small animal imaging research and clinical practice. The second review authored by Papp et al. takes on a different angle of hybrid imaging, by providing insights into big data analysis of medical image data and its potential to impact the field of personalized medicine. The third review contributed by Birkfellner et al. discusses the historical and technical development of software-based image coregistration. The fourth review article authored by Leitgeb and Baumann discusses the current state of optical coherence tomography (OCT) in precision medicine, focusing on polarization sensitive OCT, Doppler OCT and OCT angiography, OCT elastography, and spectroscopic OCT. They also consider the benefits of combining OCT with photoacoustic and non-linear optical microscopy as an example of multimodal optical imaging. Overall, these review articles provide a brief summary of the history and current status of multimodality imaging instrumentation development and medical image data analysis and processing.

The topic editors greatly appreciate significant work from contributors, reviewers, and the Frontiers supporting team led by Dr. Claudio Bogazzi. We hope the articles published in this 
Research Topic will lead to greater interest and advances in this fast-growing field to improve human healthcare.

\section{AUTHOR CONTRIBUTIONS}

All authors listed have made a substantial, direct and intellectual contribution to the work, and approved it for publication.
Conflict of Interest: The authors declare that the research was conducted in the absence of any commercial or financial relationships that could be construed as a potential conflict of interest.

Copyright $\odot 2019$ Cheng and Marriott. This is an open-access article distributed under the terms of the Creative Commons Attribution License (CC BY). The use, distribution or reproduction in other forums is permitted, provided the original author(s) and the copyright owner(s) are credited and that the original publication in this journal is cited, in accordance with accepted academic practice. No use, distribution or reproduction is permitted which does not comply with these terms. 